\title{
Conditioning Injury-Induced Spinal Axon Regeneration Requires Signal Transducer and Activator of Transcription 3 Activation
}

\author{
Jin Qiu, William B. J. Cafferty, Stephen B. McMahon, and Stephen W. N. Thompson \\ Wolfson Centre for Age-Related Diseases, Guy's, King's, and St. Thomas's School of Biomedical Science, King's College London, London SE1 1UL, United \\ Kingdom
}

\begin{abstract}
Sensory axons in the adult spinal cord do not regenerate after injury. This is essentially because of inhibitory components in the damaged CNS, such as myelin-associated inhibitors and the glial scar. However, if the sciatic nerve is axotomized before injury of the dorsal column, injured axons can regenerate a short distance in the spinal cord. Here, we show that sciatic nerve transection results in time-dependent phosphorylation and activation of the transcription factor, signal transducer and activator of transcription 3 (STAT3), in dorsal root ganglion (DRG) neurons. This effect is specific to peripheral injuries and does not occur when the dorsal column is crushed. Sustained perineural infusion of the Janus kinase 2 (JAK2) inhibitor AG490 to the proximal nerve stump can block STAT3 phosphorylation after sciatic nerve transection and results in reduced growth-associated protein 43 upregulation and compromised neurite outgrowth in vitro. Importantly, in vivo perineural infusion of AG490 also significantly attenuates dorsal column axonal regeneration in the adult spinal cord after a preconditioning sciatic nerve transection. We conclude that STAT3 activation is necessary for increased growth ability of DRG neurons and improved axonal regeneration in the spinal cord after a conditioning injury.
\end{abstract}

Key words: STAT; regeneration; condition lesion; gp130 cytokines; axon growth; sensory system

\section{Introduction}

The adult mammalian CNS does not regenerate after injury. The failure is partly attributable to an inhibitory environment in the damaged CNS, where myelin-associated inhibitors and glial scar tissues are considered as the main obstacles (Qiu et al., 2000; Schwab, 2002; Filbin, 2003; Raisman, 2004; Silver and Miller, 2004). However, recent evidence suggests that these obstacles are not insurmountable. It has been demonstrated that if intrinsic neuronal growth ability is increased, the inhibition may be partially overcome (Ramer et al., 2000; Neumann et al., 2002; Qiu et al., 2002). Thus, the outcome of CNS regeneration may represent interplay between growth ability of injured neurons and strength of inhibition within the environment.

This interplay can be illustrated by regeneration of the sensory system. Dorsal root ganglion (DRG) neurons have one axonal branch that targets peripheral tissues and another that enters the spinal cord and projects along the dorsal column. Peripheral axons readily regenerate after injury, but central axons from the same cell body do not. Interestingly, if the peripheral branch is injured first, the subsequently lesioned dorsal column axons are

Received Aug. 10, 2004; revised Dec. 12, 2004; accepted Dec. 16, 2004.

This work was supported by the Wellcome Trust (S.W.N.T.).

Correspondence should be addressed to Prof. Stephen B. McMahon, Wolfson Centre for Age-Related Diseases, King's College London, Guy's Campus, Hodgkin Building, The Wolfson Wing, London SE1 1UL, UK. E-mail: stephen.mcmahon@kcl.ac.uk.

S. W. N. Thompson's present address: School of Biological Sciences, University of Plymouth, Plymouth PL4 8AA, UK.

DOI:10.1523/JNEUROSCI.3269-04.2005

Copyright $\odot 2005$ Society for Neuroscience $\quad$ 0270-6474/05/251645-09\$15.00/0 able to regenerate a short distance (Neumann and Woolf, 1999). This is the conditioning lesion paradigm. It is believed that injuries to peripheral and central axons elicit different molecular changes in DRG neurons, and only the former increases neuronal growth ability. Reagents that augment neuronal growth can mimic the conditioning effect of peripheral injuries and induce a limited extent of axonal regeneration in the spinal cord (Neumann et al., 2002; Qiu et al., 2002).

The molecular mechanisms responsible for increased neuronal growth ability after a peripheral injury are not well understood. Previous studies have suggested a role for gp130 cytokines for which expression is induced at the lesion site after sciatic nerve transection (Banner and Patterson, 1993; Bolin et al., 1995). For example, leukemia inhibitory factor (LIF) and interleukin-6 (IL-6) can enhance neurite outgrowth of cultured DRG neurons (Cafferty et al., 2001, 2004). Peripheral nerve regeneration is impaired in mice deficient in either IL-6 or LIF (Zhong et al., 1999; Cafferty et al., 2001). Importantly, conditioning injury-induced regeneration of dorsal column axons within the CNS is also essentially compromised in IL-6 knock-out mice (Cafferty et al., 2004).

How LIF and IL-6 modulate neuronal growth is not clear. They belong to a family of cytokines that utilizes receptor complexes comprising a common signal transducing subunit, gp130, and the Janus kinase (JAK)-signal transducer and activator of transcription (STAT) signaling pathway (Aaronson and Horvath, 2002; Shuai and Liu, 2003). After axotomy, STAT3 phosphorylation is induced in facial and hypoglossal neurons but not 
in non-regenerating Clark's nucleus (Schwaiger et al., 2000). STAT3 phosphorylation is also associated with sprouting of septal neurons after entorhinal cortex lesion in adult rats (Xia et al., 2002). Moreover, STAT3 in the proximal stump is phosphorylated after sciatic nerve transection, and a wave of phosphorylated STAT3 moves along the nerve toward DRGs (Sheu et al., 2000). Here, we studied the STAT3 phosphorylation status in DRG neurons after injury and asked whether activation of the JAK-STAT signaling pathway was necessary for increased neuronal growth ability after a conditioning injury.

\section{Materials and Methods}

Animals. Animals used in this study were adult male Wistar rats (220250 g; Harlan, Bicester, Oxon, UK). All experiments involving animals were undertaken in accordance with the United Kingdom Animals (Scientific Procedure) Act 1986.

Peripheral nerve lesion and implantation of osmotic minipumps. Animals were anesthetized by inhalation of isoflurane, and unilateral sciatic nerve transection was performed at the midthigh level. For perineural infusion, the proximal stump was placed into a pliable Gortex cuff (W. L. Gore, Flagstaff, AZ) connected to an osmotic minipump $(0.25 \mu \mathrm{l} / \mathrm{h}, 4$ weeks duration; Alzet, Cupertino, CA) containing either saline or a 500 $\mu \mathrm{M}$ concentration of the JAK2 inhibitor AG490 (Calbiochem, La Jolla, CA). The osmotic minipumps were incubated at $37^{\circ} \mathrm{C}$ overnight to ensure immediate delivery after implantation. The nerve was secured to the Gortex cuff with a prolene suture (10/0; Tyco Healthcare, Basingstoke, UK). A length of flexible SILASTIC tubing (Merck, West Drayton, UK) was used to connect the Gortex cuff and the minipump, which was then placed in a pocket of skin on the back of animals.

Dorsal column crush lesion and labeling of axons. Animals were anesthetized with sodium pentobarbitone ( $40 \mathrm{mg} / \mathrm{kg}$, i.p.), and sterile precautions were used throughout. A partial laminectomy was performed at the T6 spinal cord level. A small incision was made in the dura, and lignocaine (2\%; Antigen Pharmaceuticals, Croydon, UK) was applied to the opening. The dorsal column was then crushed with fine watchmakers' forceps. The tips of the forceps were held on either side of the dorsal column, pushed $2 \mathrm{~mm}$ down into the cord, held tightly together for $10 \mathrm{~s}$, and then lifted back out of the cord. After $24 \mathrm{~d}, 3 \mu \mathrm{l}$ of cholera toxin $\beta$-subunit (CTB; $10 \mathrm{mg} / \mathrm{ml}$; List Biological Laboratories, Epsom, UK) was injected into the left sciatic nerve using a Hamilton syringe (BDH Laboratory Supplies, Poole, UK) to label myelinated fibers unilaterally in the peripheral nerve. Animals were killed after another $4 \mathrm{~d}$, and tissues were harvested and processed as described below.

Neurite outgrowth assays. Neurite outgrowth assays were performed as described previously (Cafferty et al., 2001, 2004). Briefly, L4 and L5 DRGs were removed from terminally anesthetized animals and dissociated in $0.125 \%$ collagenase (Sigma, Poole, UK) for $2 \mathrm{~h}$ at $37^{\circ} \mathrm{C}$ in a humidified chamber containing $5 \% \mathrm{CO}_{2}$. The ganglia were mechanically dissociated by gentle trituration in a solution containing $250 \mu \mathrm{g} / \mathrm{ml}$ soybean trypsin inhibitor (type II; Sigma) and $50 \mu \mathrm{g} / \mathrm{ml}$ DNase (type I; Sigma). Dissociated cells were then carefully layered onto a solution containing 15\% bovine serum albumin (Sigma) and centrifuged at 900 rpm for $6 \mathrm{~min}$. The pellet was resuspended in modified Bottenstein and Sato's medium (Cafferty et al., 2001, 2004). Dissociated DRG neurons were plated onto the Lab-TekII 8-well chamber slides (Nunc, Roskilde, Denmark) coated with $10 \mu \mathrm{g} / \mathrm{ml}$ poly-L-lysine (Sigma) followed by 10 $\mu \mathrm{g} / \mathrm{ml}$ laminin (Sigma) at a density of 100 cells per well. After culturing for $18 \mathrm{~h}$, neurons were fixed and immunostained for $\beta$-tubulin as described below. For quantification analysis, neurons with processes greater than the cell body diameter were counted as neurite-bearing cells. The length of the longest neurite of each neuron was measured from digitized images and quantified using a SigmaScan imaging analysis program (SigmaScan 4.01.003; Jandel Scientific, San Rafael, CA). The mean values of both the percentage of neurite-bearing cells and the length of the longest neurites were calculated from triplicates of experiments with four animals per experimental group.

Tissue processing and immunohistochemistry. Animals were deeply anesthetized with pentobarbitone $(80 \mathrm{mg} / \mathrm{kg}$, i.p. $)$ and perfused transcar- dially with $100 \mathrm{ml}$ of saline followed by $300 \mathrm{ml}$ of $4 \%$ paraformaldehyde in $0.1 \mathrm{M}$ phosphate buffer. Tissues were postfixed in $4 \%$ paraformaldehyde at $4^{\circ} \mathrm{C}$ for $2 \mathrm{~h}$, transferred to $20 \%$ sucrose for at least $24 \mathrm{~h}$, and then blocked in OCT embedding compound (BDH Laboratory Supplies). Longitudinal sections through the lesion site in the thoracic spinal cord and transverse sections through the dorsal column nuclei were cut on a cryostat at $20 \mu \mathrm{m}$ thickness.

Direct immunofluorescent staining of tissue sections was performed as described previously (Bradbury et al., 1999). Antibodies were diluted in a working solution of PBS containing $0.2 \%$ Triton X-100 (Sigma) and $10 \%$ normal donkey serum (The Jackson Laboratory, Bar Harbor, ME), and incubation was performed at room temperature. Details of the primary antibodies are as follows: CTB, 1:2000 (List Biological Laboratories); growth associated protein 43 (GAP43), 1:2000 (kind gift from Dr. Priestley, Queen Mary University of London, London, UK); and N52, 1:4000 (Sigma). All of the secondary antibodies (donkey anti-goat cyanine 3, donkey anti-mouse tetramethylrhodamine isothiocyanate, and donkey anti-rabbit FITC) were from The Jackson Laboratory and were used at a dilution of 1:200. Phosphorylated STAT3 was detected using an anti-phospho-STAT3 (Tyr705) antibody (1:200; Cell Signaling Technology, Beverly, MA) and an avidin-biotin-peroxidase complex reaction (Vectastain Elite ABC kit; Vector Laboratories, Burlingame, CA) according to the instructions of the manufacturer. The peroxidase complex was visualized with tyramide as the chromagen, and the nuclei were counterstained using 4',6-diamidino-2-phenylindole (DAPI; $500 \mathrm{ng} / \mathrm{ml}$; Sigma).

Cultured DRG neurons were fixed in $4 \%$ paraformaldehyde for $1 \mathrm{~h}$ at room temperature and permeabilized by incubation with ice-cold methanol for 2 min. Neurites were stained with an anti- $\beta$-tubulin primary antibody (1:5000; Sigma) for $4 \mathrm{~h}$ followed by a donkey anti-mouse FITC secondary antibody (1:200; The Jackson Laboratory) for $2 \mathrm{~h}$. Both incubations were performed at room temperature.

Quantification of dorsal column axonal growth. Images of CTB staining in serial longitudinal sections were captured at $100 \mu \mathrm{m}$ intervals from 5 $\mathrm{mm}$ caudal to $5 \mathrm{~mm}$ rostral to the lesion site. This was repeated for every fifth section, from dorsal to ventral, to provide a three-dimensional representation of the fiber growth. Images were captured by an AxioCam HRm camera (Zeiss, Oberkochen, Germany), which were collaged and imported to the SigmaScan. Collaged images were converted into the gray-scale representation, and a threshold level was set that was kept constant among all sections. A strip of fixed width $(400 \mu \mathrm{m})$ along the dorsal column $(\sim 7 \mathrm{~mm})$ was analyzed for the number of pixels exceeding the threshold. The fluorescence, as measured by number of pixels above a defined threshold, was then plotted against the distance to the lesion epicenter.

\section{Results}

\section{Sciatic nerve transection induces persistent STAT3 phosphorylation in DRG neurons in vivo}

It has been established that the gp 130 cytokines IL-6 and LIF play an important role in peripheral nerve regeneration after injury (Zhong et al., 1999; Cafferty et al., 2001, 2004). They use a common signaling pathway that involves recruitment and activation of the JAK-STAT signaling cascade. The presence of phosphorylated STAT (pSTAT) in the nucleus is considered a molecular signature for gp130 receptor activation. Phosphorylation of one member of the STAT family of transcription factors, STAT3, has been associated with nerve injuries (Schwaiger et al., 2000; Sheu et al., 2000; Xia et al., 2002), but it is not yet known whether it is present in DRG neurons after sciatic nerve transection. To detect STAT3 phosphorylation, we used an antibody that specifically recognizes the phosphorylated residue tyrosine 705 .

In all experiments, pSTAT3 immunoreactivity was analyzed in L4 and L5 DRGs after left sciatic nerve transection at the midthigh level or a bilateral dorsal column crush. The proportion of nuclear profiles expressing pSTAT3 in injured ganglia was compared with that in naive or corresponding contralateral uninjured DRGs. In DRG sections from naive rats and all contralat- 

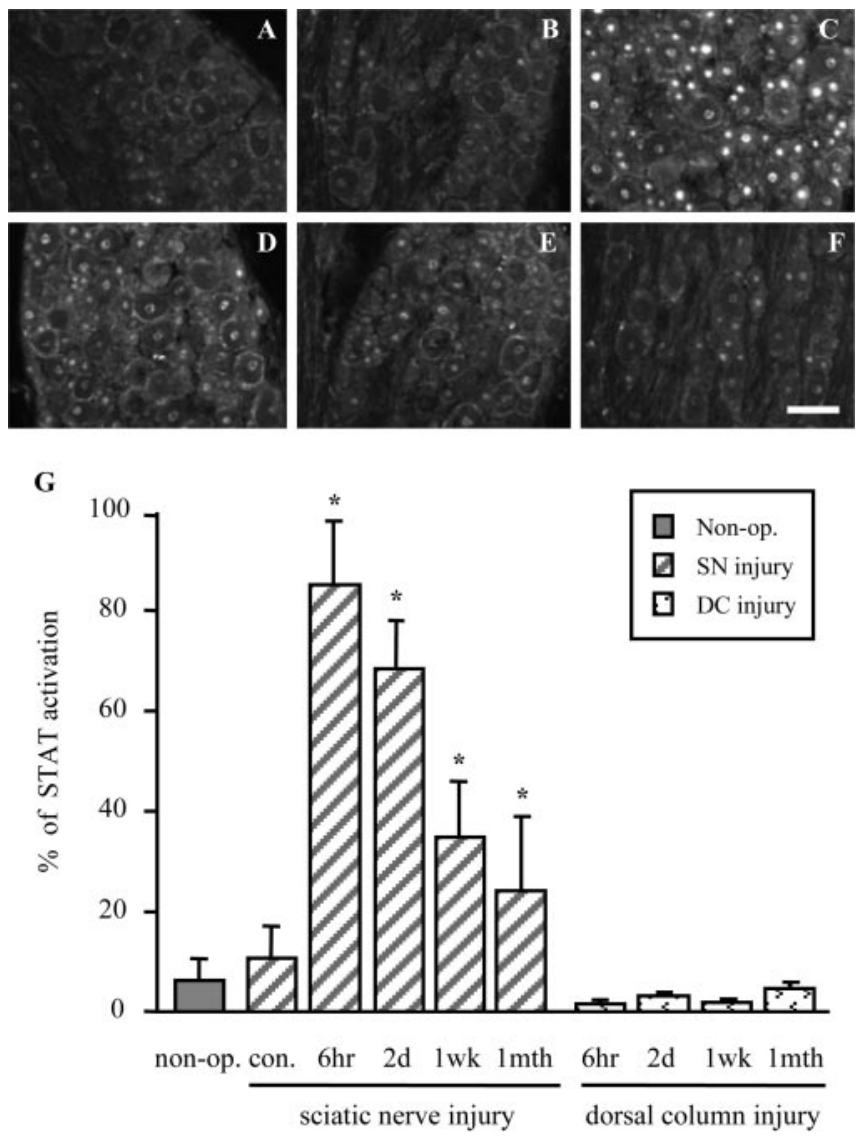

Figure 1. Time course of STAT3 phosphorylation in response to sciatic nerve transection and dorsal column crush. PSTAT3 immunoreactivity was analyzed in L4 and L5 DRGs after left sciatic nerve transection at the midthigh level or bilateral dorsal column crush. The percentage of pSTAT3-positive cells was calculated against the total number of neurons with DAPI staining. In DRG sections from naive animals $(A)$ and all contralateral ganglia $(B)$, pSTAT3 staining was minimal (7.3 \pm 4.3 and $10.7 \pm 6.2 \%$, respectively). Six hours $(C), 2 \mathrm{~d}(D), 1$ week $(E)$, and 1 month $(F)$ after sciatic nerve transection, there was a significant increase in the proportion of nuclear profiles expressing pSTAT3 compared with contralateral DRGs $(90.2 \pm 12.7,68.4 \pm$ 9.5, $34.9 \pm 11.2$, and $24.1 \pm 6.2 \%$ compared with $10.7 \pm 6.2 \% ; p<0.05$ in all cases; Student's $t$ test; $n=5$ ). This elevation in PSTAT was not observed at the same time points after dorsal column (DC) crush (data not shown). Scale bar, $50 \mu \mathrm{m}$. G, Quantification of the proportion of nuclear profiles expressing pSTAT3 in DRGs after left sciatic nerve (SN) transection and bilateral dorsal column crush. Con, Control; Non-op., nonoperated. ${ }^{*} p<0.05$.

eral sections, pSTAT3 was essentially absent (Fig. $1 A, B$ ). However, $6 \mathrm{~h}$ after sciatic nerve transection, we observed a highly increased level of pSTAT3 in the nucleus of ipsilateral DRG neurons (Fig. 1C). This increased level of pSTAT3 declined with time but remained significantly elevated for up to 1 month after injury (Fig. $1 D-F$ ). In contrast, there was no significant changes in the pSTAT levels in DRG neurons at any time points studied after a bilateral dorsal column crush (Fig. 1G).

All DRG sections were counterstained with DAPI to reveal nuclear profiles (data not shown). The proportion of DRG neurons expressing pSTAT3 at each time point after sciatic nerve axotomy was expressed as a percentage of the total number of nuclear profiles examined after DAPI staining. Six h, $2 \mathrm{~d}$, and 1 week after injury, there was a significant increase in the proportion of nuclear profiles expressing pSTAT3 in ipsilateral DRGs compared with the contralateral control $(90.2 \pm 12.7,68.4 \pm 9.5$, and $34.9 \pm 11.2 \%$ compared with $10.7 \pm 6.2 \%$, respectively; $p<$ 0.05 in all cases; Student's $t$ test; $n=5$ ). One month after sciatic axotomy, the proportion of nuclear profiles expressing pSTAT3 was still significantly higher from the control value $(24.1 \pm 6.2$ and $10.7 \pm 6.2 \%$, respectively; $p<0.05$; Student's $t$ test; $n=5$ ). At all time points studied, the proportion of DRG neurons expressing pSTAT after dorsal column lesion remained comparable with that from naive animals $(1.5 \pm 0.5,3.2 \pm 0.2,1.7 \pm 0.6$, and $4.8 \pm 1.1 \%$ compared with $6.3 \pm 4.3 \%$, respectively). Together, these data show that sciatic nerve transection, but not dorsal column lesion, results in rapid and persistent STAT3 phosphorylation in the DRG neurons.

\section{Axotomy-induced STAT3 phosphorylation is blocked by perineural infusion of the JAK2 inhibitor AG490}

The gp130 cytokine signaling pathway has been implicated in improved growth capacity of DRG neurons after a conditioning lesion (Cafferty et al., 2001, 2004; Liu and Snider, 2001), but direct evidence is still lacking. We conjectured that if a pathway is necessary for inducing a particular biological effect, then blocking the pathway at any point should diminish or at least reduce the very effect it induces. It has been suggested that, after a peripheral injury, STAT3 is phosphorylated by JAK tyrosine kinases at the lesion site, and pSTAT is then retrogradely transported to the cell body and translocated into the nucleus (Schweizer et al., 2002). Under these circumstances, blocking phosphorylation of STAT at the injury site should prevent its nuclear localization and downstream biological consequences of the pathway. Here, we used the JAK2 inhibitor AG490 to block activation of the JAKSTAT signaling pathway at the site of nerve injury. AG490 has been widely used to block JAK2 and STAT3 activation in culture (Liu and Snider, 2001; Ruscher et al., 2002; Sandberg and Sayeski, 2004).

Immediately after sciatic nerve axotomy, the proximal stump of the cut sciatic nerve was secured within a pliable Gortex cuff that was connected to an osmotic minipump via a silicon cannula. The pump was filled with either saline or AG490 (500 $\mu \mathrm{M})$ delivered at a rate of $0.5 \mu \mathrm{l} / \mathrm{h}$. It has been reported that $50 \mu \mathrm{M}$ AG490 is effective in blocking the JAK-STAT signaling pathway in vitro (Liu and Snider, 2001; Ruscher et al., 2002; Sandberg and Sayeski, 2004). According to our previous experience of pharmacological studies, in vivo studies normally require a dose $10-100$ times higher than the one used in vitro, although it is likely to cause toxic effects at the higher end. Continuous delivery of AG490 for 4 weeks to the proximal nerve stump significantly reduced the proportion of nuclear profiles expressing pSTAT3 compared with the saline treatment group (Fig. 2A-C). Quantification analysis showed that there was a $38.0 \%$ reduction in STAT3 phosphorylation in the injured, ipsilateral DRG neurons when AG490 was delivered to the proximal nerve stump immediately after injury $(26.3 \pm 6.4$ and $16.3 \pm 5.1 \%$, respectively; $p<$ 0.05 , Student's $t$ test; $n=5$ ) (Fig. $2 D$ ). Neither AG490 nor saline had any effect on the basal level of pSTAT3 in contralateral DRGs (Fig. 2D). These data suggest that perineural infusion of AG490 at the time of injury is able to block STAT phosphorylation and activation in DRG neurons as a result of sciatic nerve transection.

\section{Axotomy-induced GAP43 upregulation in DRG neurons in vivo is inhibited by JAK2 inhibition}

We next examined the effect of inhibition of STAT3 activation on a neuronal growth indicator. It has been demonstrated that, contrary to central lesions such as those of dorsal column axons, sciatic nerve transection results in an increased expression of a number of growth-associated proteins such as GAP43 and cytoskeleton-associated protein 23 (CAP23) in DRG neurons (Schreyer and Skene, 1991, 1993; Chong et al., 1994; Andersen 

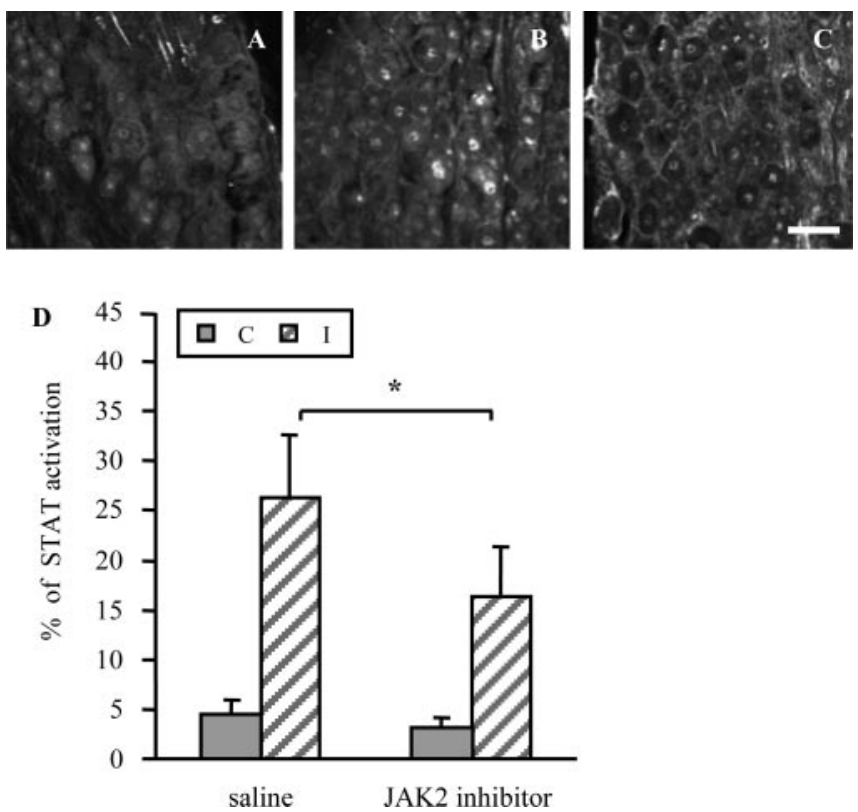

Figure 2. STAT3 phosphorylation induced by sciatic nerve transection is blocked by perineural infusion of the JAK2 inhibitor AG490. PSTAT3 immunoreactivity was analyzed in L4 and L5 DRGs after left sciatic nerve transection at the midthigh level and continuous perineural infusion

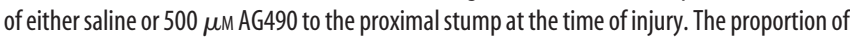
nuclear profiles expressing pSTAT3 in ipsilateral ganglia was calculated against the total number of neurons with DAPI staining. A, Contralateral DRGs treated with either saline or AG490 expressed a minimal amount of pSTAT. Continuous infusion of AG490 ( $C$ ) to the proximal nerve stump for 4 weeks significantly reduced the proportion of nuclear profiles expressing pSTAT3 compared with the saline treatment group $(B)(16.3 \pm 5.1$ compared with $26.3 \pm 6.4 \%$, respectively; $p<0.05$; Student's $t$ test; $n=5)$. Scale bar, $50 \mu \mathrm{m}$. D, Quantification of pSTAT3 staining in contralateral [C] and ipsilateral [I] L4 and L5 DRGs after left sciatic nerve transection and continuous infusion of either saline or a JAK2 inhibitor, $\mathrm{AG490.}{ }^{*} p<0.05$.

and Schreyer, 1999). It is suggested that high levels of these proteins are associated with increased neuronal growth capacity and, therefore, are generally regarded as growth indicators. Here, we analyzed GAP43 expression in DRG neurons after sciatic nerve transection and perineural infusion of either saline or the JAK2 inhibitor AG490.

GAP43 is normally expressed in a small percentage of DRG neurons in naive animals. After sciatic nerve injury, GAP43 expression in ipsilateral DRG neurons is significantly elevated, predominantly in large- and medium-sized neuronal populations, but that in the contralateral counterpart remains at the basal control level. Perineural infusion of either saline or AG490 at the injury site had no effect on GAP43 expression in contralateral DRGs (data not shown). Four weeks after sciatic nerve axotomy and perineural infusion of saline, the proportion of neuronal profiles expressing GAP43 was significantly elevated in ipsilateral DRGs compared with contralateral controls $(57.5 \pm 7.2$ and $22.2 \pm 5.6 \%$, respectively) (Fig. $3 A, B, J$ ). Perineural infusion of AG490 significantly attenuated this increase in GAP43 expression in ipsilateral DRGs after injury ( $42.9 \pm 9.9$ compared with $57.5 \pm$ $7.2 \% ; p<0.05$; Student's $t$ test; $n=5$ ) (Fig. $3 B, C, J$ ).

GAP43 expression was examined more closely within neuronal populations by costaining DRG neurons with an antibody against neurofilament-200 (NF200), a commonly used marker of large-diameter DRG neurons that project their central axons along dorsal column in the spinal cord. The proportion of neuronal profiles expressing NF200 in contralateral and ipsilateral DRGs was comparable after sciatic nerve transection and did not change significantly after perineural infusion of either saline or
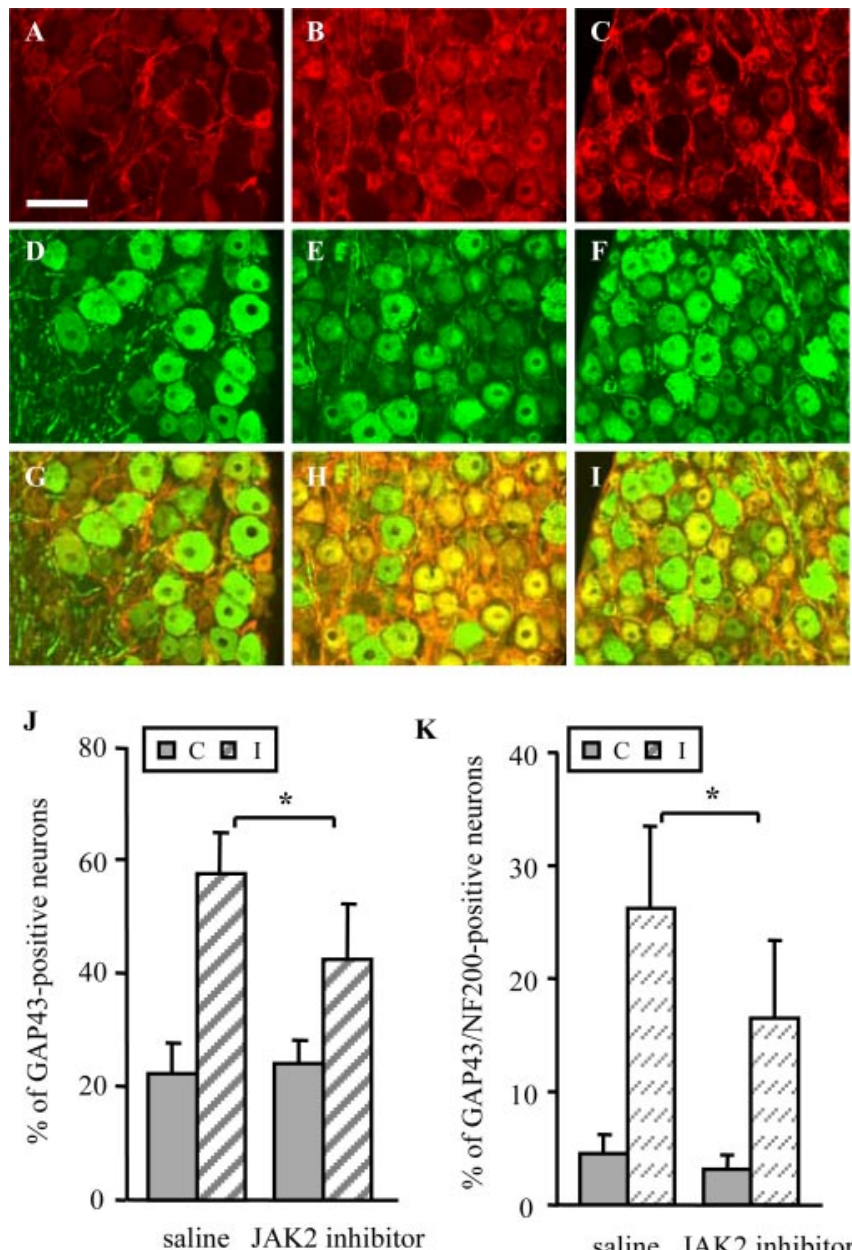

Figure 3. Increased expression of GAP43 induced by sciatic nerve transection is blocked by JAK2 inhibition. Expression of GAP43 $(A-C)$ and NF200 $(D-F)$ was analyzed in contralateral $(A$, $D, G)$ and ipsilateral $(B, C, E, F, H, I) L 4$ and $L 5$ DRGs after left sciatic nerve transection at the midthigh level and continuous perineural infusion of either saline $(B, E, H)$ or $500 \mu \mathrm{m} A G 490(C$, $F, I)$ to the proximal stump at the time of injury. Overlay of the GAP43 and NF200 immunoreactivity is shown in G-I. GAP43 expression is significantly elevated in ipsilateral ganglia after sciatic nerve transection $(B)$, and the effect is reduced by perineural infusion of $A G 490$ ( $C$ ). The proportion of neuronal profiles coexpressing GAP43 and NF200 (yellow profiles) is also significantly reduced after perineural infusion of $\operatorname{AG} 490(H, I)$. Scale bar, $50 \mu \mathrm{m}$. J, Quantification of the proportion of neuronal profiles expressing GAP43 in contralateral [C] and ipsilateral [I] L4 and L5 DRGs after left sciatic nerve transection and continuous infusion of either saline or the JAK2 inhibitor AG490. K, Quantification of the proportion of neuronal profiles coexpressing NF200 and GAP43 in contralateral [C] and ipsilateral [I] L4 and L5 DRGs after left sciatic nerve transection and continuous infusion of either saline or a JAK2 inhibitor, AG490. ${ }^{*} p<0.05$.

AG490 (Fig. 3D-F). However, the proportion of neuronal profiles expressing both GAP43 and NF200 was significantly reduced in ipsilateral DRGs treated with AG490 compared with that in the saline control group (Fig. $3 G-I, J$ ).

Quantification studies show that only a small proportion of uninjured DRG neurons expressed both NF200 and GAP43 $(4.6 \pm 1.6 \%)$ (Fig. 3G,J). After sciatic nerve transection, this number increased by approximately fivefold in ipsilateral DRG neurons $(26.2 \pm 7.2 \%$ ) (Figure $3 \mathrm{H}, \mathrm{J})$. Perineural infusion of AG490 led to a significantly smaller percentage of neurons with elevated GAP43 expression within the subpopulation of NF200positive neurons $(16.5 \pm 6.9$ compared with $26.2 \pm 7.2 \%$; $p<$ 0.05 ; Student's $t$ test; $n=5$ ) (Figure $3 H-J$ ). These data indicate that perineural infusion of the JAK2 inhibitor AG490 is able to 
block upregulation of a growth indicator, GAP43, in NF200expressing DRG neurons.

\section{Conditioning lesion-induced neurite outgrowth in vitro is attenuated by JAK2 inhibition in vivo}

After sciatic nerve transection, DRG neurons increase their growth propensity and can extend much longer neurites in vitro. This is known as the conditioning effect. Recently, we and others suggested that the gp130 cytokine signaling pathway might be important in mediating this injury-induced axonal growth response (Cafferty et al., 2001, 2004; Liu and Snider, 2001). We have shown that improved growth of DRG neurons after a conditioning injury is diminished both in vitro and in vivo in IL-6 and LIF knock-out mice (Cafferty et al., 2001, 2004). To assess whether the gp130 signaling pathway is important in the conditioning effect, we blocked activation of the pathway by perineural infusion of the JAK2 inhibitor AG490 at the injury site immediately after sciatic nerve transection. After 1 week of sustained treatment, we measured neurite extension of DRG neurons in vitro and compared neuronal growth ability after different treatments.

Dissociated adult DRG neurons from uninjured, contralateral ganglia had minimal ability to extend neurites during the $18 \mathrm{~h}$ culture period in the absence of serum or growth factors (Fig. $4 A, C)$. In saline-treated animals, ipsilateral DRG neurons were able to extend long neurites with an average length of $333.4 \pm$ $19.7 \mu \mathrm{m}$ (Fig. $4 B, E$ ). This improved neuronal growth in culture after a conditioning lesion was significantly reduced when AG490 was infused perineurally throughout the week after injury $(179.6 \pm 22.3$ compared with $333.4 \pm 19.7 \mu \mathrm{m} ; p<0.05$; Student's $t$ test; $n=9$ ) (Fig. $4 D, E$ ). This could result from an effect on either initiation of neurite outgrowth or process elongation, which are recognized as two distinct phases of neuronal growth. We found that AG490 did not affect the percentage of neurons that bear neurites $(73.7 \pm 13.9$ and $64.9 \pm 11.9 \%$, respectively) (Fig. 4F), indicating that AG490 might attenuate neurite elongation.

These data indicate that perineural infusion of an inhibitor of the gp130 signaling cascade at the time of sciatic nerve injury significantly attenuates improved growth ability of DRG neurons in vitro as a result of conditioning injury. The observation that only neurite length is affected, but not the percentage of neuritebearing cells, suggests that AG490 appears to block neurite elongation rather than initiation of outgrowth.

Conditioning lesion-induced spinal axon regeneration in vivo is inhibited by JAK2 inhibition

Our present data are consistent with previous findings (Cafferty et al., 2001, 2004) that suggest a role for the gp130 signaling pathway in increasing neuronal growth ability after a peripheral injury. We reported previously that improved regeneration of dorsal column axons after a conditioning lesion is essentially compromised in IL-6 knock-out mice (Cafferty et al., 2004). Here, we asked whether blocking activation of the JAK-STAT pathway could also affect the conditioning effect on dorsal column axonal regeneration in adult spinal cord.

A conditioning injury to the sciatic nerve has been shown to enhance regeneration of the subsequently lesioned dorsal column axons in the adult spinal cord (McQuarrie and Grafstein, 1973; Richardson and Issa, 1984; Richardson and Verge, 1986, 1987; Neumann and Woolf, 1999). This conditioning effect depends on the length of time between the peripheral and central lesions, and the optimal effect is achieved when the peripheral
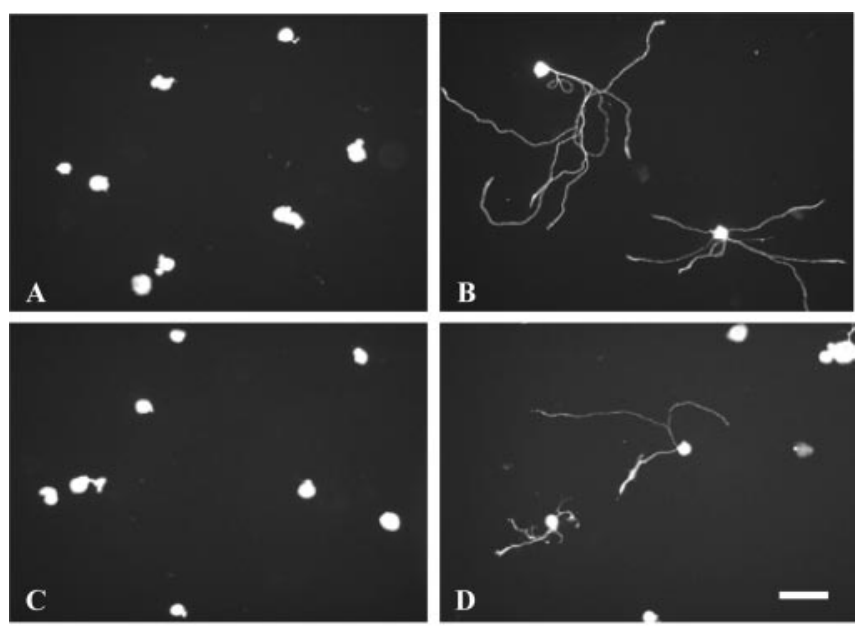

E

F
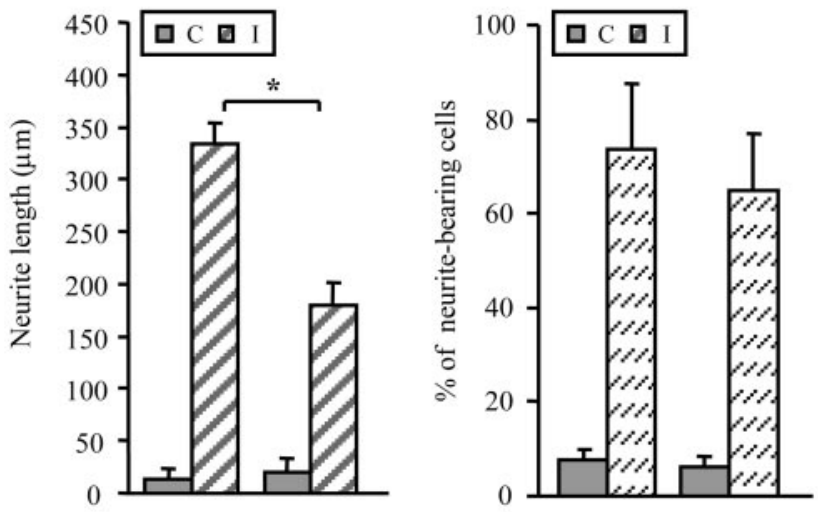

saline JAK2 inhibitor

saline JAK2 inhibitor

Figure 4. JAK2 inhibition at the time of injury blocks the conditioning effect of sciatic nerve transection on neurite outgrowth in vitro. Neurite outgrowth analysis was performed with contralateral $(A, C)$ and ipsilateral $(B, D)$ L4 and L5 DRG neurons after left sciatic nerve transection at the midthigh level and continuous perineural infusion of either saline $(A, B)$ or $500 \mu \mathrm{m}$ AG490 $(C, D)$ to the proximal stump for 1 week. Dissociated DRG neurons were cultured for $18 \mathrm{~h}$ and then fixed and immunostained for $\beta$-tubulin. Scale bar, $100 \mu \mathrm{m}$. Perineural infusion of 500 $\mu \mathrm{M} \mathrm{AG} 490$ at the time of injury significantly reduced neurite elongation $(E)$ within the culture period (179.6 \pm 22.3 compared with $333.4 \pm 19.7 \%$; ${ }^{*} p<0.05$; Student's $t$ test; $n=9$ ) but had no effect on the percentage of neurite-bearing cells $(F)(64.9 \pm 1.9$ compared with $73.7 \pm$ $13.9 \%)$.

injury precedes the central lesion by 1 week (Neumann and Woolf, 1999). To test whether inhibiting the JAK-STAT signaling pathway can block the conditioning effect on regeneration in vivo, the left sciatic nerve was transected, and either saline or AG490 was infused into the proximal stump by an osmotic minipump, and the infusion was performed for the following 4 weeks. One week after sciatic nerve transection and infusion, the dorsal column axons were crushed bilaterally at the T6 spinal cord level, and animals were allowed to recover for 4 weeks after the crush injury. Four days before they were killed, all animals received a unilateral injection of the transganglionic tracer CTB to their left sciatic nerve.

It is widely accepted that the forceps crush of spinal nerves at the thoracic level would most likely be able to completely destroy the gracile fasciculus, which is the bundle of fibers labeled by CTB injection into the sciatic nerve. To confirm this, brainstem sections were stained with an antibody against CTB. In uninjured animals, numerous axon terminals could be seen in the gracile nucleus as revealed by the CTB immunoreactivity (Fig. 5A). A 
bilateral crush of the dorsal column at T6 severed the entire dorsal column projection pathway, which resulted in a loss of CTB staining in the brainstem (Fig. 5B). These data suggest that dorsal column lesion was complete, and any labeled fibers at and rostral to the injury site were regenerating axons.

In animals that received sciatic nerve transection and saline infusion $7 \mathrm{~d}$ before bilateral T6 dorsal column crush, thick bundles of CTB-labeled fibers were found in the injured adult spinal cord immediately caudal to the lesion (Fig. 5C). Many axons were able to grow into or around the injury site that was enriched in potent inhibitory molecules from damaged myelin and glial scar tissues. Some nerve fibers managed to extend processes rostral to the lesion epicenter. These findings are consistent with what has been reported previously (Richardson and Issa, 1984; Neumann and Woolf, 1999; Cafferty et al., 2004). In contrast, in animals that received sciatic nerve transection and AG490 infusion $7 \mathrm{~d}$ before dorsal column crush, very few CTB-labeled fibers were observed at the injury site or immediately caudal to it (Fig. 5D). Most of the fibers appeared rather dispersed and tangled. In none of the animals examined $(n=5)$ were axonal fibers observed rostral to the lesion site. This apparent difference in the number of CTB-labeled axons around the lesion site was not caused by a difference in labeling efficiency between treatment groups as indicated by staining of CTB-labeled axons at the lumbar level (Fig. 5E,F).

The degree of regeneration was quantified by measuring the fluorescence of CTB-labeled fibers and then plotting the values against the distance from the lesion epicenter. In both treatment groups, the fluorescence as measured by pixels above a defined threshold drops sharply when approaching the lesion site (Fig. 6). There was a significant difference in the fluorescence around the lesion site between the saline and AG490 treatment groups. In saline-treated animals, the fluorescence diminished around the lesion epicenter with a small percentage of florescence reaching beyond. In contrast, in animals treated with AG490, this decline was much more dramatic, and little fluorescence was detectable within $2 \mathrm{~mm}$ caudal to the lesion site. To assess whether AG490 completely abolished the conditioning effect, we studied dorsal column axonal regeneration in the absence of a conditioning lesion. There were many more CTB-labeled spinal axons caudal to the lesion site after a conditioning injury (either with saline or AG490 infusion) than dorsal column injury alone (supplementary figure, available at www.jneurosci.org as supplemental material). Labeling efficiency in all experimental groups was comparable. With regard to axon regeneration rostral to the injury site, the effect of AG490 on the conditioning effect appeared complete. The possibility remains that dieback of axons might be restricted by conditioning injuries, even in the presence of AG490. Additional studies are required to investigate possible mechanisms that do not involve activation of the JAK-STAT signaling pathways. Together, these data demonstrate that perineural infusion of an inhibitor of the gp130 signaling pathway at the time of peripheral nerve injury is sufficient to prevent improved regeneration of dorsal column axons in the adult spinal cord.

\section{Discussion}

This study shows for the first time that STAT3, a downstream effector molecule of gp130 cytokines, is phosphorylated in DRG neurons after sciatic nerve transection but not dorsal column axon crush. Phosphorylation of STAT3 correlates with enhanced neuronal growth ability. Importantly, when a JAK2 inhibitor, AG490, is used to block activation of JAK-STAT3 signaling pathway in response to peripheral axotomy, improved regeneration of dorsal column axons is diminished. This is direct in vivo evidence that an injury-induced signaling pathway is crucial in increasing neuronal growth ability after a conditioning lesion. 


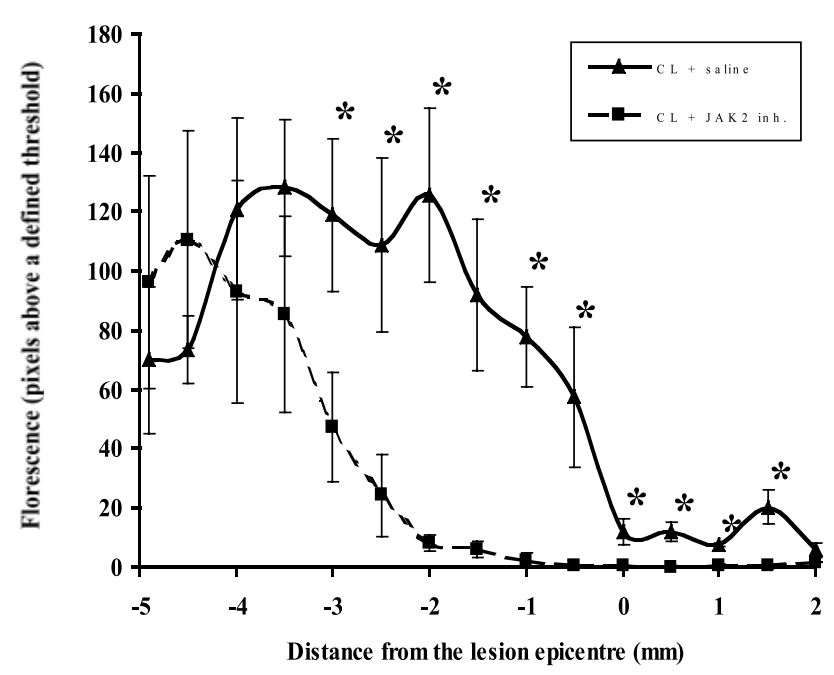

Figure 6. Quantification of CTB-labeled regenerating axons in longitudinal spinal cord sections from animals that received dorsal column lesion (CL) 1 week after left sciatic nerve transection and continuous infusion of either saline or the JAK2 inhibitor (inh.) AG490. Fluorescent intensity was measured at defined intervals caudal and rostral to the lesion epicenter ${ }^{*} p<$ 0.05; Student's $t$ test; $n=5$ ).

It has been postulated that inflammatory responses after a peripheral injury might be beneficial to axonal regeneration ( $\mathrm{Lu}$ and Richardson, 1991; Richardson and Lu, 1994). Many studies have focused on gp130 cytokines because they are important in regulating injury-mediated cellular responses in the periphery. Both LIF and IL-6 are strongly upregulated after sciatic nerve transection, and some injury responses are lost in mice deficient in either cytokine (Kurek et al., 1996; Reichert et al., 1996; Zhong et al., 1999; Inserra et al., 2000; Cafferty et al., 2001, 2004). For example, both $\mathrm{LIF}^{-/-}$and IL-6 ${ }^{-/-}$mice display reduced efficiency of peripheral nerve regeneration (Zhong et al., 1999; Cafferty et al., 2001). Improved growth of DRG neurons both in vitro and in vivo after sciatic nerve transection is diminished without IL-6 or LIF (Inserra et al., 2000; Cafferty et al., 2001, 2004). Although these studies are consistent with the hypothesis that LIF and IL-6 are important in regulating neuronal growth, these data are open to alternative interpretations, because both cytokines are crucial in PNS development and IL-6 is also important in astrocytic responses in the CNS. More definitive evidence requires inducible knock-out models of either cytokines or their common signaling receptor, gp 130. Alternatively, as presented in this study, effects of blocking the shared JAK-STAT signaling pathway could provide more definitive evidence. Our study shows that when activation of STAT3, the main downstream effector molecule of LIF and IL-6 (Levy and Darnell, 2002; Herrmann et al., 2003), is blocked, neuronal responses to sciatic nerve transection are essentially compromised. Importantly, severed dorsal column axons fail to regenerate after a conditioning injury. We conclude that JAK2-STAT3 signaling pathway is important in increasing growth ability of DRG neurons after peripheral injury. This is consistent with previous findings that LIF and IL-6 can stimulate neuronal growth in vitro (Cafferty et al., 2001, 2004) and that improved growth of DRG neurons in vitro after a conditioning lesion can be blocked by AG490 (Liu and Snider, 2001).

Our finding that the JAK2-STAT3 pathway is crucial for improved neuronal growth after sciatic nerve transection is important for two reasons. First, this demonstrates directly that a path- way triggered preferentially by a peripheral injury is necessary for increased neuronal growth ability. Many studies have documented that peripheral and central injuries induce different molecular changes in the neuronal cell body (Broude et al., 1997; Herdegen et al., 1997; Herdegen and Leah, 1998; Schwaiger et al. 2000; Sheu et al., 2000; Snider et al., 2002), which is partly responsible for different regeneration outcomes in the respective nervous system. However, most of these studies are merely descriptive and correlative, and only a couple of studies provide functional evidence to support the conjecture. Bomze et al. (2001) showed that when both GAP43 and CAP23 are overexpressed, spinal cord regeneration is significantly augmented. Raivich et al. (2004) demonstrated that a nervous system-specific deficiency in c-Jun expression markedly impedes regeneration of motor neurons after injury. It has been reported that axotomy induces STAT phosphorylation in facial and hypoglossal neurons but not in the non-regenerating Clarke's nucleus neurons (Schwaiger et al., 2000). Although STAT3 phosphorylation has been detected in transected sciatic nerve (Sheu et al., 2000), our study demonstrates for the first time that peripheral but not central injuries induce STAT3 activation in DRG neurons. More importantly, STAT3 activation correlates with increased neuronal growth ability, and blockade of this activation pathway diminishes injury-induced improved regenerative potential in vitro and in vivo.

Second, our study presents the first direct evidence that the JAK-STAT pathway is important in nerve regeneration. Although first implicated in the immune system, the JAK-STAT pathway is also involved in wide-ranging biological functions such as embryonic development, homeostasis, cell growth, and survival (Aaronson and Horvath, 2002; Levy and Darnell, 2002; Schweizer et al., 2002; Shuai and Liu, 2003). In the nervous system, the JAK-STAT3 pathway is important in astrocyte differentiation in development and astrocytic responses after CNS injury (Sun et al., 2001; Xia et al., 2002). STAT3 activation in septal neurons is associated with collateral sprouting after entorhinal cortex lesion (Xia et al., 2002). STAT3 is also activated in facial motoneurons after peripheral nerve transection, which is necessary for the survival of these axotomized neurons (Haas et al., 1999; Schweizer et al., 2002). In this study, we have extended the repertoire of the biological functions of STAT3 in the nervous system by establishing its role in neuronal growth and regeneration.

Previous studies indicate that two mechanisms might be responsible for STAT3 phosphorylation and subsequent translocation into the nucleus of DRG neurons after sciatic nerve transection (Hendry et al., 1992; Curtis et al., 1994; Murphy et al., 1995, 1999; Thompson et al., 1997; Sheu et al., 2000; Hanz et al., 2003; Kirsch et al., 2003). First, the JAK-STAT signaling pathway might be activated at the injury site as a result of gp130 cytokines released after axotomy, and pSTAT is then retrogradely transported to the cell body. This is consistent with the evidence that, after sciatic nerve transection, pSTAT3 is present in both proximal and distal stumps, and a wave of pSTAT3 moves along the nerve toward the DRG (Sheu et al., 2000). When retrograde transport is blocked by colchicine, STAT3 phosphorylation induced in facial motoneurons after axotomy is suppressed (Kirsch et al., 2003). Translocation of pSTAT into the nucleus is mediated by the nuclear localization signal (NLS) in the protein (Levy and Darnell, 2002; Pires-daSilva and Sommer, 2003; Shuai and Liu, 2003). Retrograde transport of pSTAT depends on binding of NLS to the motor protein, dynein, and adding synthetic NLS at the injury site delays peripheral nerve regeneration and reduces the condi- 
tioning effect on axonal growth of DRG neurons (Hanz et al., 2003). Thus, pSTAT might be one of the positive injury signals that is induced at the lesion site and transported to the cell body, where it elicits cellular changes such as gene expression.

Second, the JAK-STAT pathway might be activated in the cell body by gp130 cytokines either produced in the ganglia or retrogradely transported from the injury site. LIF and CNTF (but not IL-6) are induced at the injury site after sciatic nerve transection and then retrogradely transported to the DRGs (Hendry et al., 1992; Curtis et al., 1994; Thompson et al., 1997). One-third of lumbar DRG neurons begin to synthesize IL-6 after axotomy (Murphy et al., 1995), which depends on mast cell-related retrograde signals from the injury site (Murphy et al., 1999). It has yet to be determined whether AG490, infused to the proximal stump, is confined to the injury site or is transported by an unknown mechanism to the cell body. It is conceivable that activation of JAK-STAT pathway might be an immediate early response after sciatic nerve transection, which is important in subsequent signaling cascades. Attenuation of this process might inhibit local cytokine production and retrograde transport of injury-induced molecules. Additional studies are required to differentiate these possibilities. Regardless of the mechanism whereby AG490 reduces the pSTAT3 levels in DRGs, our study demonstrates that activation of JAK2-STAT3 pathway is necessary for increased neuronal growth ability after a conditioning injury.

It has been reported that cAMP signaling pathway is important for neuronal growth (McQuarrie et al., 1977; Qiu et al., 2000, 2002; Cai et al., 2001). The cAMP levels in adult DRGs are intrinsically low but significantly elevated $1 \mathrm{~d}$ after sciatic nerve transection (Neumann et al., 2002; Qiu et al., 2002). The conditioning effect can be blocked by inhibitors of protein kinase A, a downstream effector molecule of cAMP, and direct injection of cAMP into the DRGs can mimic the effect of peripheral lesion and promote dorsal column regeneration in the adult spinal cord (Neumann et al., 2002; Qiu et al., 2002). It is not known how the cAMP levels are upregulated in DRGs or whether the upregulation requires retrograde signals from the injury site. Here, we identified a novel signaling pathway in the conditioning lesion paradigm. It would be interesting to determine whether there are molecular cross-talks between cAMP and JAK-STAT pathways after peripheral injury and whether activation of both pathways could further augment regeneration of dorsal column axons. Preliminary evidence demonstrates that cAMP can stimulate synthesis of IL-6 mRNA in DRG neurons in culture (M. Filbin, personal communications). It remains to be seen whether cAMP can also induce IL-6 and other cytokine production in vivo and whether activation of JAK-STAT pathway after sciatic nerve transection depends on elevation of the cAMP levels in DRGs.

\section{References}

Aaronson DS, Horvath CM (2002) A road map for those who don't know JAK-STAT. Science 296:1653-1655.

Andersen LB, Schreyer DJ (1999) Constitutive expression of GAP-43 correlates with rapid, but not slow regrowth of injured dorsal root axons in the adult rat. Exp Neurol 155:157-164.

Banner LR, Patterson PH (1993) Major changes in the expression of the mRNAs for cholinergic differentiation factor/leukemia inhibitory factor and its receptor after injury to adult peripheral nerves and ganglia. Proc Natl Acad Sci USA 91:7109-7113.

Bolin LM, Verity AN, Silver JE, Shooter EM, Abrams JS (1995) Interleukin-6 production by Schwann cells and induction in sciatic nerve injury. J Neurochem 64:850-858.

Bomze HM, Bulsara KR, Iskandar BJ, Caroni P, Skene JH (2001) Spinal axon regeneration evoked by replacing two growth cone proteins in adult neurons. Nat Neurosci 4:38-43.
Bradbury EJ, Khemani S, Von R, King, Priestley JV, McMahon SB (1999) NT-3 promotes growth of lesioned adult rat sensory axons ascending in the dorsal columns of the spinal cord. Eur J Neurosci 11:3873-3883.

Broude E, McAtee M, Kelley MS, Bregman BS (1997) c-Jun expression in adult rat dorsal root ganglion neurons: differential response after central or peripheral axotomy. Exp Neurol 148:367-377.

Cafferty WB, Gardiner NJ, Gavazzi I, Powell J, McMahon SB, Heath JK, Munson J, Cohen J, Thompson SW (2001) Leukemia inhibitory factor determines the growth status of injured adult sensory neurons. J Neurosci 21:7161-7170.

Cafferty WBJ, Das P, Gardiner NJ, Qiu J, Thompson SW (2004) Conditioning injury-induced spinal axon regeneration fails in interleukin-6 knockout mice. J Neurosci 24:4432-4443.

Cai D, Qiu J, Cao Z, McAtee M, Bregman BS, Filbin MT (2001) Neuronal cAMP controls the developmental loss in ability of axons to regenerate. J Neurosci 21:4731-4739.

Chong MS, Reynolds ML, Irwin N, Coggeshall RE, Emson PC, Benowitz LI, Woolf CJ (1994) GAP-43 expression in primary sensory neurons following central axotomy. J Neurosci 14:4375-4384.

Curtis R, Scherer SS, Somogyi R, Adryan KM, Ip NY, Zhu Y, Lindsay RM, DiStefano PS (1994) Retrograde axonal transport of LIF is increased by peripheral nerve injury: correlation with increased LIF expression in distal nerve. Neuron 12:191-204.

Filbin M (2003) Myelin-associated inhibitors of axonal regeneration in the adult mammalian CNS. Nat Rev Neurosci 4:703-713.

Haas CA, Hofmann HD, Kirsch M (1999) Expression of CNTF/LIFreceptor components and activation of STAT3 signaling in axotomized facial motoneurons: evidence for a sequential postlesional function of the cytokines. J Neurobiol 41:559-571.

Hanz S, Perlson E, Willis D, Zheng JQ, Massarwa R, Huerta JJ, Koltzenburg M, Kohler M, van-Minnen J, Twiss JL, Fainzilber M (2003) Axoplasmic importins enable retrograde injury signaling in lesioned nerve. Neuron 40:1095-1104.

Hendry IA, Murphy M, Hilton DJ, Nicola NA, Bartlett PF (1992) Binding and retrograde transport of leukemia inhibitory factor by the sensory nervous system. J Neurosci 12:3427-3434.

Herdegen T, Leah JD (1998) Inducible and constitutive transcription factors in the mammalian nervous system: control of gene expression by Jun, Fos and Krox, and CREB/ATF proteins. Brain Res Brain Res Rev 28:370-490.

Herdegen T, Skene P, Bahr M (1997) The c-Jun transcription factor-bipotential mediator of neuronal death, survival and regeneration. Trends Neurosci 20:227-231.

Herrmann A, Sommer U, Pranada AL, Giese B, Kuster A, Haan S, Becker W, Heinrich PC, Muller-Newen G (2003) STAT3 is enriched in nuclear bodies. J Cell Sci 117:339-349.

Inserra MM, Yao M, Murray R, Terris DJ (2000) Peripheral nerve regeneration in interleukin 6-deficient mice. Arch Otolaryngol Head Neck Surg 126:1112-1116.

Kirsch M, Terheggen U, Hofmann HD (2003) Ciliary neurotrophic factor is an early lesion-induced retrograde signal for axotomized facial motoneurons. Mol Cell Neurosci 24:130-138.

Kurek JB, Austin L, Cheema SS, Bartlett PF, Murphy M (1996) Upregulation of leukaemia inhibitory factor and interleukin-6 in transected sciatic nerve and muscle following denervation. Neuromuscul Disord 6:105-114.

Levy DE, Darnell Jr JE (2002) Stats: transcriptional control and biological impact. Nat Rev Mol Cell Biol 3:651-662.

Liu RY, Snider WD (2001) Different signaling pathways mediate regenerative versus developmental sensory axon growth. J Neurosci 21:RC164(1-5).

Lu X, Richardson PM (1991) Inflammation near the nerve cell body enhances axonal regeneration. J Neurosci 11:972-978.

McQuarrie IG, Grafstein B (1973) Axon outgrowth enhanced by a previous nerve injury. Arch Neurol 29:53-55.

McQuarrie IG, Grafstein B, Gershon MD (1977) Axonal regeneration in the rat sciatic nerve: effect of a conditioning lesion and of dbcAMP. Brain Res 132:443-453.

Murphy PG, Grondin J, Altares M, Richardson PM (1995) Induction of interleukin-6 in axotomized sensory neurons. J Neurosci 15:5130-5138. Murphy PG, Borthwick LS, Johnston RS, Kuchel G, Richardson PM (1999) 
Nature of the retrograde signal from injured nerves that induces interleukin-6 mRNA in neurons. J Neurosci 19:3791-3800.

Neumann S, WoolfCJ (1999) Regeneration of dorsal column fibers into and beyond the lesion site following adult spinal cord injury. Neuron 23:83-91.

Neumann S, Bradke F, Tessier-Lavigne M, Basbaum AI (2002) Regeneration of sensory axons within the injured spinal cord induced by intraganglionic cAMP elevation. Neuron 34:885-893.

Pires-daSilva A, Sommer RJ (2003) The evolution of signalling pathways in animal development. Nat Rev Genet 4:39-49.

Qiu J, Cai D, Filbin M (2000) Glial inhibition of nerve regeneration in the mature mammalian CNS. Glia 29:166-174.

Qiu J, Cai D, Dai H, McAtee M, Hoffman PN, Bregman BS, Filbin MT (2002) Spinal axon regeneration induced by elevation of cyclic AMP. Neuron 34:895-903.

Raisman G (2004) Myelin inhibitors: does NO mean GO? Nat Rev Neurosci 5:157-161.

Raivich G, Bohatschek M, Da Costa C, Iwata O, Galiano M, Hristova M, Nateri AS, Makwana M, Riera-Sans L, Wolfer DP, Lipp HP, Aguzzi A, Wagner EF, Behrens A (2004) The AP-1 transcription factor c-Jun is required for efficient axonal regeneration. Neuron 43:57-67.

Ramer MS, Priestley JV, McMahon SB (2000) Functional regeneration of sensory axons into the adult spinal cord. Nature 403:312-316.

Reichert F, Levitzky R, Rotshenker S (1996) Interleukin 6 in intact and injured mouse peripheral nerves. Eur J Neurosci 8:530-535.

Richardson PM, Issa VM (1984) Peripheral injury enhances central regeneration of primary sensory neurones. Nature 309:791-793.

Richardson PM, Lu X (1994) Inflammation and axonal regeneration. J Neurol 242:S57-S60.

Richardson PM, Verge VM (1986) The induction of a regenerative propensity in sensory neurons following peripheral axonal injury. J Neurocytol 15:585-594.

Richardson PM, Verge VM (1987) Axonal regeneration in dorsal spinal roots is accelerated by peripheral axonal transection. Brain Res 411:406-408.

Ruscher K, Freyer D, Karsch M, Isaev N, Megow D, Sawitzki B, Priller J, Dirnagl U, Meisel A (2002) Erythropoietin is a paracrine mediator of ischemic tolerance in the brain: evidence from an in vitro model. J Neurosci 22:10291-10301.

Sandberg EM, Sayeski PP (2004) Jak2 tyrosine kinase mediates oxidative stress-induced apoptosis in vascular smooth muscle cells. J Biol Chem 279:34547-34552.

Schreyer DJ, Skene JH (1991) Fate of GAP-43 in ascending spinal axons of DRG neurons after peripheral nerve injury: delayed accumulation and correlation with regenerative potential. J Neurosci 11:3738-3751.

Schreyer DJ, Skene JH (1993) Injury-associated induction of GAP-43 expression displays axon branch specificity in rat dorsal root ganglion neurons. J Neurobiol 24:959-970.

Schwab ME (2002) Repairing the injured spinal cord. Science 295:1029-1031.

Schwaiger FW, Hager G, Schmitt AB, Horvat A, Hager G, Streif R, Spitzer C, Gamal S, Breuer S, Brook GA, Nacimiento W, Kreutzberg GW (2000) Peripheral but not central axotomy induces changes in Janus kinases (JAK) and signal transducers and activators of transcription (STAT). Eur J Neurosci 12:1165-1176.

Schweizer U, Gunnersen J, Karch C, Wiese S, Holtmann B, Takeda K, Akira S, Sendtner M (2002) Conditional gene ablation of Stat3 reveals differential signaling requirements for survival of motoneurons during development and after nerve injury in the adult. J Cell Biol 156:287-297.

Sheu JY, Kulhanek DJ, Eckenstein FP (2000) Differential patterns of ERK and STAT3 phosphorylation after sciatic nerve transection in the rat. Exp Neurol 166:392-402.

Shuai K, Liu B (2003) Regulation of JAK-STAT signalling in the immune system. Nat Rev Immunol 3:900-911.

Silver J, Miller JH (2004) Regeneration beyond the glial scar. Nat Rev Neurosci 5:146-156.

Snider WD, Zhou FQ, Zhong J, Markus A (2002) Signaling the pathway to regeneration. Neuron 35:13-16.

Sun Y, Nadal-Vicens M, Misono S, Lin MZ, Zubiaga A, Hua X, Fan G, Greenberg ME (2001) Neurogenin promotes neurogenesis and inhibits glial differentiation by independent mechanisms. Cell 104:365-376.

Thompson SW, Vernallis AB, Heath JK, Priestley JV (1997) Leukaemia inhibitory factor is retrogradely transported by a distinct population of adult rat sensory neurons: co-localization with trkA and other neurochemical markers. Eur J Neurosci 9:1244-1251.

Xia XG, Hofmann HD, Deller T, Kirsch M (2002) Induction of STAT3 signaling in activated astrocytes and sprouting septal neurons following entorhinal cortex lesion in adult rats. Mol Cell Neurosci 21:379-392.

Zhong J, Dietzel ID, Wahle P, Kopf M, Heumann R (1999) Sensory impairments and delayed regeneration of sensory axons in interleukin-6deficient mice. J Neurosci 19:4305-4313. 